\title{
A proteção auditiva utilizada pelos militares do Exército brasileiro: há efetividade?
}

\author{
Hearing protection used by the military \\ of the Brazilian army: is it effective?
}

Eduardo Borba N eves ${ }^{1}$

M arcia Soalheiro ${ }^{2}$
${ }^{1}$ Pós-graduação da Escola de Aperfeiçoamento deOficiais do Exército. Av. Duquede Caxias 2.947/BI 6 /101, Vila Militar. 21615-220 Rio de Janeiro RJ.

borbaneves@hotmail.com

${ }^{2}$ Centro deEstudos da Saúde do Trabalhador eEcologia

Humana, Escola Nacional de SaúdePública, Fundação Oswaldo Cruz.
Abstract This study aims to identify the noise exposure and verify if the hearing protection used by these workers is adjusted for the exposure to the impact noise during the activities of Basic Shot Instruction in the Brazilian Army produced by Light Automatic Fusils- FAL, caliber $7.62 \mathrm{~mm}$. This study can be considered analytical, since it seeks to establish causal relations among the diverse variables involved. It was used the qualitative approach, in the mapping of the working process; and also the quantitative approach in order to analyze the data in relation to this process. It was possi ble to have a close estimate of the reality on the basis of data collection triangulation, to include: systematic observation, half structured interview and experimental exercise. Based on the analyses, it can be stated these workers are exposed to a noise which ranges from 147.3 to 171 $\mathrm{dB}$. Therefore, it can be concluded that the $3 \mathrm{M}$ 1100 hearing protection, which is largely used by military, do not provide noise reduction, since its reducing value corresponds to $\mathrm{NRRs}$ at $12 \mathrm{~dB}$. Thus, there is no effectiveness in hearing protection for the soldiers.

Key words Impact noise, Occupational noise, Military, Worker health
Resumo Neste estudo, buscou-se identificar a exposição sonora e verificar se a proteção auditiva utilizada por esses trabalhadores é adequada para a exposição ao ruído de impacto, durante as atividades de Tiro de Instrução Básico realizadas no Exército Brasileiro, produzidos pelo Fuzil Automático Leve- FAL, calibre 7,62 mm. 0 presente estudo caracteriza-se como analítico, uma vez que busca estabelecer relações causais entre as diversas variáveis envolvidas. U tilizou-se da abordagem qualitativa, no mapeamento do processo de trabalho; eda abordagem quantitativa na analise dos dados em relação a esse processo. Foi possível uma aproximação da realidade a partir de uma triangulação de técnicas de coleta de dados, a saber: observação sistemática; entrevista semi-estruturada e exercício experimental. Diante das análises realizadas, pode- se garantir queesses trabalhadores estão expostos a um N PS mínimo de $147,3 \mathrm{~dB}$ durante as atividades de tiro, podendo esse valor atingir $171 \mathrm{~dB}$. Assim, entende-sequea real redução do ruído, oferecida pelo protetor 1100 da $3 \mathrm{M}$, principal modelo utilizado pelos militares, cujo valor de atenuação auditiva corresponde a N RRsf de $12 \mathrm{~dB}$, não caracteriza efetividade na proteção auditiva para os militares.

Palavras-chave Ruído de impacto, Ruído ocupacional, M ilitar, Saúde do trabalhador 
Introdução

A exposição a níveis elevados de ruído por um longo período de tempo podeacarretar comprometimentos físicos, mentais e sociais nos indivíduos. Entre esses, o mais definido e quantificado é o dano ao sistema auditivo, que depende de fatores tais como frequência, intensidade, duração, ritmo, tempo de exposição e susceptibilidadeindividual ${ }^{1}$.

0 dano auditivo produzido pela exposição a níveis elevados de pressão sonora pode ser classificado em três tipos: trauma acústico, perda auditiva temporária e perda auditiva permanente. 0 trauma acústico éirreversível, permanente e ocorre quando o ouvido humano é exposto ao ruído de impacto, com intensidade sonora da ordem de $120 \mathrm{~dB}$ ou superior. Esta carga sonora produzirá, na cóclea, lesões intensas, como ruptura da membrana basilar e desorganização abrupta dos tecidos e células ciliadas. Clinicamente, apresenta-se por perda auditiva do tipo neurossensorial, imediata e permanente, uni ou bilateral, com presença de zumbidos constantes$^{2}$. Já a análise ocupacional, preconizada pela Portaria no 19, de 9 de abril de 1998, que versa sobre as diretrizes e parâmetros mínimos para avaliação e acompanhamento da audição de trabalhadores expostos a níveis de pressão sonora elevados, permite classificá-la em sugestiva de perda auditiva induzida por ruído por níveis de pressão sonora elevados e através da evolução da patologia em desencadeamento ou, até mesmo, agravamento da perda auditiva induzida por níveis de pressão sonora elevados ${ }^{3}$.

Oliveira atribui o trauma acústico ao som explosivo instantâneo com pico de pressão sonora que excede $140 \mathrm{~dB}$. Estes níveis sonoros al cançam as estruturas do ouvido interno, excedendo os limites de elasticidade dos tecidos, produzindo a ruptura do órgão de Corti, sendo este desligado da membrana basilar que, por sua vez, é destruída esubstituída por tecido epitelial escamoso, restabelecendo a integridade do comportamento do fluído da escala média (M B) e órgão de Corti ${ }^{4}$.

Segundo Spoendlin, citado por Costa et al. ${ }^{5}$, as células ciliadas externas têm grande atividade mecânica e são pouco protegidas pelas células de sustentação, degeneram-se primeiro e em mai or quantidade; porém, com o avanço da lesão, deterioram-se, depois, as células ciliadas internase com elas as fibras nervosas, sempre em menor extensão. Em fases mais adiantadas, degeneramse também as células de sustentação, despovoando e colabando todo o órgão de Corti, de for- ma que os ruídos de impacto produzem lesões nas estruturas do órgão de Corti, através de ação mecânica, com consequente processo degenerativo e os ruídos contínuos e prolongados originam alterações predominantemente por exaustão metabólica 5 .

De acordo com Bohne e Slepecky, também citados por Costa et al. ${ }^{5}$, a área mais alterada localiza-sena espira basal da cóclea, no setor correspondente às frequências em torno de $4.000 \mathrm{~Hz}$ e, como consequência dessas lesões, surgem outras alterações, como o recrutamento, que se reflete na intolerância por sons intensos. Isso reduz sensivelmente o campo dinâmico da audição, provoca dificuldades para reconhecer sons complexos, como os da fala, em condições ambientais desfavoráveis à escuta (ruído de fundo, fala competitiva, reverberação das paredes, sinais com emissão defeituosa) e apresenta dificuldades para localizar as fontes sonoras.

0 ruído deimpacto édefinido na N R15 como aquele que apresenta picos de energia acústica de duração inferior a um segundo, a intervalos superiores a um segundo, com limite de tolerância de $130 \mathrm{~dB}$, ou $120 \mathrm{~dB}(\mathrm{C})$, utilizando-se a constante de tempo "fast". Esta mesma norma orienta queníveis de ruído contínuo ou intermitente, acima de $115 \mathrm{~dB}(\mathrm{~A})$, constituem-secomo risco grave ou iminente e que não são permitidos em situações em que não haja a proteção adequada 6 .

O National Institute for Occupational Safety and $\mathrm{H}$ ealth (NIOSH) recomenda quea avaliação do ruído de impacto seja realizada utilizando-se a medida do nível equivalente e considera como uma área que ainda deve ser objeto de maiores pesquisas ${ }^{7}$. Segundo $M$ endes ${ }^{8}$, ainda não há consenso estabelecido para a avaliação deste tipo de ruído, por conta do risco que proporciona. Há evidências científicas indicadoras de que os efeitos da exposição ao ruído deimpacto não seajustam à regra da igual energia. Outras variáveis teriam influência como os parâmetros amplitude, duração, tempo de subida, número de impactos, taxa de repetição e fator de crista. Desta forma, a presença de ruído de impacto combinada com o ruído contínuo teria um efeito sinérgico ${ }^{9}$. Por outro lado, há também pesquisas que afirmam que a presença de ruído de impacto apresenta apenas um efeito aditivo, significando quea avaliação pelo nível equivalente na escala $\mathrm{A}$ é suficiente para predizer o risco $0^{6}$.

A N orma de HigieneO cupacional 01 da Fundacentro ${ }^{10}$ estabelece que o trabalhador não deve ficar exposto a níveis de pressão sonora superiores a 140 dB. Essa mesma publicação apresenta 
uma fórmula, que foi utilizada neste trabalho, para o cálculo da quantidade de pulsos de impacto a que o trabalhador pode ficar exposto em cada jornada de trabal ho, de acordo com a magnitude do pulso ${ }^{10}$.

Um estudo anterior, realizado com 97 militares expostos ao ruído intenso em exercícios de tiros, identificou que $38,1 \%$ apresentaram quadro otológico sugestivo de perda auditiva induzida por ruído. A perda auditiva encontrada foi mais intensa em função da idade e do tempo de serviço, tendo sido observado que, dos militares examinados, 64,59\% não utilizaram proteção auditiva adequada nos exercícios de tiro ${ }^{9}$. A Revista Brasileira de Otorrinolaringologia atribui ao rifleautomático (classificação genérica do Fuzil Automático Leve - FAL), um nível de pressão sonora (NPS) de $171 \mathrm{~dB}^{11}$.

Em virtude da existência de inúmeros trabaIhos publicados sobre perda auditiva em militares $^{12-15}$, buscou-se, neste estudo, identificar a exposição sonora e verificar se a proteção auditiva utilizada por esses trabalhadores é adequada para a exposição ao ruído de impacto, durante as atividades de Tiro de Instrução Básico realizadas no Exército Brasileiro, produzidos pelo Fuzil Automático Leve (FAL), calibre 7,62 mm.

\section{Procedimentos metodológicos}

0 presente estudo caracteriza-se como analítico, uma vez que busca estabelecer relações causais entre as diversas variáveis envolvidas. Utilizouse da abordagem qualitativa, em sua dimensão mais objetiva ${ }^{16}$, no mapeamento do processo de trabal ho; e da abordagem quantitativa na análise dos dados em relação a esse processo. Foi possível uma aproximação da realidade a partir de uma triangulação de técnicas de coleta de dados, a saber: observação sistemática; entrevista semiestruturada e exercício experimental.
A observação sistemática foi real izada durante a realização do Tiro de Instrução Básico (TIB), em cinco unidades do exército. As entrevistas foram do tipo semiestruturada com roteiro único, cujo objetivo foi mapear e entender o processo de trabalho. A entrevista semiestruturada é um instrumento que possibilita a coleta de informações objetivas e, mais do que isto, permite captar a subjetividade embutida em valores, atitudes e opiniões ${ }^{17}$. Foram entrevistados militares, distribuídos por cinco organizações militares (OM).

A seleção dos sujeitos foi balizada pela participação dos indivíduos nos exercícios de tiro de fuzil, particularmente no Tiro de Instrução Básico (TIB). A amostragem utilizada, 24 sujeitos, de cunho intencional, é compatível com a abordagem qualitativa e foi considerada suficiente, na medida em que a riqueza do material se mostrou adequada para o mapeamento do processo de trabalho dos militares em relação à atividade de tiro e, além disso, os discursos se tornavam recorrentes, o que é denominado ponto de redundância ${ }^{18}$.

0 exercício experimental foi realizado pelos próprios pesquisadores e consistiu na medição do ruído produzido por um fuzil nas condições descritas no Quadro 1.

Utilizou-se munição do tipo comum, do lote 2004, da Companhia Brasileira de Cartuchos (CBC). Os equipamentos utilizados para a medição foram: o medidor de nível sonoro Bruel \& Kjær, Tipo 2236, № de série 2100587, № Certificado/Ano 0032/2006; termohigrômetro Vaisala tipo H M 34, N o de sérieX5110004, № Certificado/Ano 1600/2004; calibrador sonoro Bruel \& Kjær, Tipo 4231, № de série 2376729, № Certificado/Ano 1815/2005; todos do Laboratório de Ensaios Acústicos da Divisão de Acústica e Vibrações do Instituto Nacional de Metrologia, N ormalização eQ ualidadeI ndustrial (Inmetro).

A medição do ruído de impacto foi realizada no circuito de compensação $C$ e circuito de res-

Quadro 1. Condições de execução do experimento.

\begin{tabular}{|c|c|c|c|c|}
\hline \multirow[b]{3}{*}{ Arma } & \multicolumn{4}{|c|}{ Local } \\
\hline & \multicolumn{2}{|c|}{ Estande } & \multicolumn{2}{|c|}{ Campo aberto } \\
\hline & $d B(A)$ & $\mathrm{dB}(\mathrm{C})$ & $d B(A)$ & $\mathrm{dB}(\mathrm{C})$ \\
\hline Fuzil (FAL) 7,62mm M 964 & $\begin{array}{l}\text { Posição: de pé } \\
5 \text { disparos }\end{array}$ & $\begin{array}{l}\text { Posição: de pé } \\
5 \text { disparos }\end{array}$ & $\begin{array}{l}\text { Posição: de pé } \\
5 \text { disparos }\end{array}$ & $\begin{array}{l}\text { Posiçãa: de pé } \\
5 \text { disparos }\end{array}$ \\
\hline
\end{tabular}

Temperatura durantea realização do experimento: $31^{\circ} \mathrm{C}$ 
posta rápida (FAST), indicada para as medidas de ruídos de impacto, de acordo com a NR 15, em seu Anexo II ${ }^{6}$. Foram coletadas três medidas do ruído para se obter uma média, respeitando 0 intervalo de trinta segundos entre cada medida. Em todas as medidas, a função M AX do aparelho foi ativada, fixando-se assim, no visor, 0 pico máximo do ruído atingido durante cada tiro realizado.

0 estande de tiro possuía estrutura de alve naria, sem qualquer recurso que promovesse a dissi pação do som, e cobertura em telhas de barro. 0 ruído foi medido em um dos boxes de tiro, cujas medidas estão apresentadas na Figura 1. A medida, em campo aberto, foi realizada com um afastamento mínimo de $15 \mathrm{~m}$ de qualquer superfície que pudesse influenciar o processo.

0 estudo seguiu os aspectos éticos recomendados pela Resolução no 196/96 do CNS sobre pesquisa envolvendo seres humanos e teve seu protocolo aprovado pelo Comitê de Ética em pesquisa do Instituto de Estudos em SaúdeColetiva da Universidade Federal do Rio de Janeiro (CEP/IESC).

\section{O Tiro deInstrução Básico}

O Tiro de Instrução Básico (TIB) éum exercício de tiro realizado por todos os soldados do Exército, quando da sua formação, durante o primeiro ano na Força. Essa atividade é normatiza- da pelas Instruções Gerais de Tiro com o Armamento do Exército (IGTAEx), que descreve de maneira detalhada cada atividade de tiro realizada durante 0 ano de instrução ${ }^{19}$.

Das unidades pesquisadas, somente duas possuíam estande compatível com a padronização técnica para a realização do TIB; as demais o fizeram em estandes deoutras unidades. Das cinco atividades de tiro acompanhadas, duas foram em estandes com box de tiro coberto e três com box de tiro em campo aberto.

A segurança, em seu sentido mais amplo, é um item que recebe grande atenção nas atividades que foram observadas, as ações durante essas atividades são executadas somente após a ordem do instrutor ou condutor; porém, com relação à proteção auditiva, foi observado que não existem procedimentos de instrução de uso, sendo este de uso puramente opcional. A proteção auditiva para essa atividade deve prover não só a atenuação dos ruídos, mas também a possibilidade de entendimento das ordens e orientações durante as atividades realizadas.

Em linhas gerais, os dispositivos adotados nessas atividades são basicamente os mesmos. Ao chegar ao estande, os instrumentos são divididos por séries, de acordo com a capacidade do estande. Para cada box, são designados um atirador eum municiador; os demais ficam em torno de dez metros da retaguarda da linha de tiro, aguardando o término das séries anteriores. Portanto, em relação à exposição ao ruído, todos os
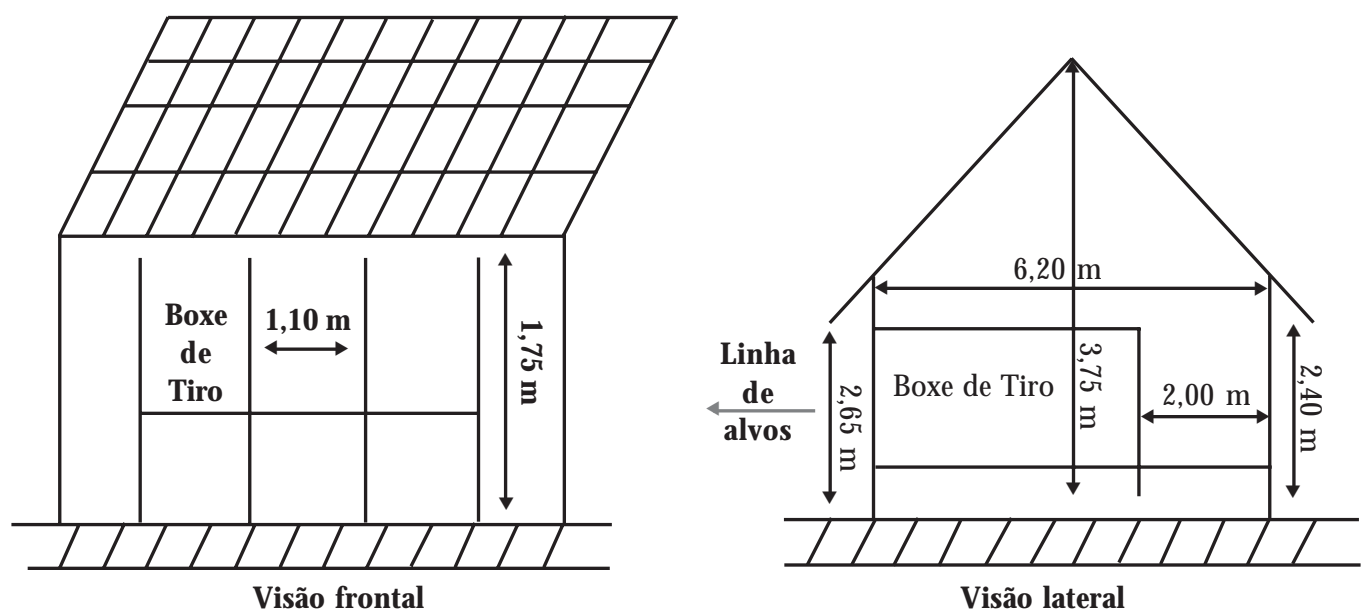

Figura 1. Estande do experimento. 
soldados e instrutores permanecem no estande até o término de todas as séries.

As condições de execução dos exercícios observados estão descritas no Quadro 2.

Pode-se constatar, no quadro acima, que a soma dos tiros previstos para cada militar é de 25 tiros. A quantidade de indivíduos que realizaram o TIB nas unidades pesquisadas está descrita no Quadro 3.

De acordo com o quadro acima, podemos calcular a quantidade de tiros e pulsos de impacto realizados em cada jornada diária, por unidade, multiplicando o numero de tiros previstos (25 tiros) pelo número de indivíduos no dia. Obtivemos um intervalo entre 1.750 e 2.900 pulsos diários.

\section{A proteção auditiva}

0 protetor auditivo é a solução mais comum e usada em nível mundial nos casos em queas técnicas de controle de ruído não são disponíveis de imediato ou até que ações sejam tomadas para a redução de ruído nas fontes, trajetórias ou receptores até o limite permitido ${ }^{20}$.

Segundo Berger ${ }^{21}$, existem quatro caminhos pelos quais o som pode atingir a orelha, mesmo estando ocluída por um protetor auricular: as passagens de ar, em que se não houver uma adequada adaptação do protetor à orel ha, podeocorrer uma redução da atenuação da ordem de 5 a $15 \mathrm{~dB}$, em uma ampla banda de frequências; a vibração do protetor, em que, no caso de protetores de inserção, pode ocorrer vibração em razão da flexibilidadedo canal auditivo limitando a atenuação nas baixas frequências; a transmissão pelo material, que está relacionada à massa, rigidez e vedação e a condução óssea e pelos tecidos.

O tempo de uso do protetor para que ocorra efetividadena atenuação também precisa ser considerado, uma vez que o uso intermitente deste dispositivo provocará uma redução significativa em sua eficiência ${ }^{22}$. Para níveis de exposição a partir de $100 \mathrm{~dB}(\mathrm{~A})$, é aconsel hado o uso de dupla-proteção, salientando-se que há somente um acréscimo de 5 a $10 \mathrm{~dB}(\mathrm{~A})$ de atenuação, conforme Nixon e Berger, citados pelo NIOSH ${ }^{22}$.

Os protetores se dividem, fundamentalmente, da seguinte forma: passivos, que são os mais comuns e não possuem nenhum mecanismo especial para a redução do ruído; não-lineares, em que a atenuação oferecida é proporcional ao nível deruído existenteno ambiente, apresentando especial interesse em ambientes com predominância de ruído intermitente e de impacto e dotados de redução ativa de ruído, em queégerado um som sensivelmente idêntico, porém em fase oposta ${ }^{23}$.

Com relação à atenuação oferecida por um protetor auditivo, existem diversos métodos para estimar a proteção. 0 mais utilizado é o Noise Reducing Rating (NRR); porém, essemétodo não leva em consideração a colocação do equipamento pelo trabalhador, o que pode afetar consideravelmente o nível de proteção, por conta de faIhas na adaptação ao pavilhão auditivo.

Segundo o boletim técnico da 3M , o protetor auditivo modelo 1100 foi testado de acordo com a norma ANSI S3.19/1974 (American National

Quadro 2. Condições de execução do Tiro de Instrução Básico (TIB).

\begin{tabular}{|c|c|c|l|}
\hline \multicolumn{4}{|c|}{ Condições de execução } \\
\hline Luz & Posição & $\begin{array}{c}\text { Tiros por } \\
\text { homem }\end{array}$ & M unição \\
\hline Diurno & Deitado, apoiado; & 4 & Comum \\
& Deitado & 4 & \\
& De joelhos, apoiado; & 4 & \\
& De pé, modificado; & 2 & \\
& De pé, modificado; & 2 & \\
& De pé, modificado; & 3 & Traçante \\
\hline \multirow{5}{*}{ Noturno } & De pé, modificado; & 3 & \\
& De joelhos, apoiado & 3 & \\
& & \multicolumn{2}{c}{} \\
\hline
\end{tabular}

Quadro 3. Quantidade de indivíduos que realizaram o TIB.

\begin{tabular}{|l|l|l|l|l|}
\hline Unidade & \multicolumn{1}{|c|}{$1^{\text {ㅇ dia }}$} & \multicolumn{1}{c|}{ 20 dia } & 30 dia & 4 dia \\
\hline 1 & 116 indivíduos & 116 indivíduos & & \\
\hline 2 & 70 indivíduos & 70 indivíduos & 70 indivíduos & \\
\hline 3 & 100 indivíduos & 100 indivíduos & & \\
\hline 4 & 95 indivíduos & 95 indivíduos & 95 indivíduos & 80 indivíduos \\
\hline 5 & 73 indivíduos & 73 indivíduos & 74 indivíduos & \\
\hline
\end{tabular}


Standard Institute) pelo laboratório Michael \& Associated Inc., Estados Unidos, sendo submetido ao ensaio de ouvido real (método subjetivo), tendo sido obtido o Nível de Redução de Ruído (NRR) de $29 \mathrm{~dB}^{24}$. Entretanto, alguns estudos sugerem que seja feita uma redução de $50 \%$ no valor de NRR e esta redução tem sido uma recomendação do NIOSH desde junho/1998, por se considerar que 0 valor de NRR foi obtido com pessoas em laboratório, pelo fato de que no ambiente real de trabalho, a atenuação obtida pelo usuário de proteção auditiva é menor. Nesse mesmo documento, há uma recomendação para que esse protetor não seja utilizado para ruídos acima de $114 \mathrm{~dB}(\mathrm{~A})$.

Em 1997, foi aprovada uma nova norma para os ensaios dos protetores auditivos ANSI 12.61997, na qual existem dois métodos; um deles é denominado método $B$, que consiste na colocação do protetor pelo ouvinte. Nesta norma, os ouvintes que participam nos ensaios do laboratório não devem ter experiência do uso do protetor, eles somente lêem as instruções dos fabricantes antes do teste. 0 executor do teste não pode colocar o protetor ou modificar a colocação ou, ainda, conversar com os ouvintes. Também os ouvintes participam poucas vezes nos testes e são trocados por outros novos ouvintes. Portanto, os ouvintes representam os trabalhadores no campo do mundo real. Os valores de atenuação, desvio padrão eNRRsf ( colocação do protetor pelo próprio indivíduo) obtidos são os mais próximos possíveis dos valores obtidos no mundo real do campo ${ }^{20}$.

0 boletim técnico supracitado também apresenta o resultado desse novo método de teste, para o mesmo protetor, realizado de acordo com a norma ANSI S12.6/1997 - método B - M étodo do Ouvido Real - Colocação pelo O uvinte, pelo laboratório LARI (UFSC/SC), no qual foi obtido o Nível de Redução de Ruído NRRsf de 12 dB. Entretanto, também há uma recomendação de que para este valor de N RRsf não ultrapasse níveis de pressão sonora acima de $98 \mathrm{~dB}(\mathrm{~A})^{24}$.
A legislação brasileira através da $\mathrm{N}$ orma $\mathrm{Re}$ gulamentadora $n^{\circ} 6$ trata dos equipamentos de proteção individual (EPI) e prescreve a obrigatoriedade da empresa em fornecer aos trabalhadores o protetor auricular para trabalhos realizados em locais em que o nível de ruído seja superior ao estabelecido na NR-15, anexos I ell ${ }^{6}$.

Os níveis de pressão sonora que são emitidos nas instruções de tiro do Exército se enquadram nesse caso; porém, como as relações trabalhistas das Forças Armadas são baseadas no Estatuto dos Militares ${ }^{25}$ e não na Consolidação das Leis Trabal histas (CLT), o Exército indica a utilização da proteção auditiva nessas instruções, mas não a fornece ${ }^{26}$. Portanto, a proteção auditiva utilizada pelos soldados ocorre por livre escolha, isto é, os próprios soldados éque devem escolher a proteção auditiva e providenciar sua aquisição com recursos próprios.

Nesta operação, pôde-se constatar: uso de protetor auditivo do tipo de inserção, descartável, modelo 1100 da 3M , uso de al godão na forma encontrada nas farmácias ou, então, nenhuma proteção auditiva.

\section{Resultados do experimento}

Os equipamentos utilizados para a medição do ruído emitido por Fuzil Automático Leve (FAL), calibre 7,62 mm foram calibrados e aferidos segundo os critérios do IN M ETRO, instituição governamental credenciada ehabilitada para tal. Os níveis de pressão sonora produzidos pelo tiro de fuzil, em todas as condições avaliadas, excederam a capacidade de medição do aparelho, apresentando resultados com a expressão overload, que significa que o nível de pressão sonora (NPS) real está acima dos níveis registrados pelo aparelho.

As médias dos resultados encontrados nas duas situações em queforam avaliadas estão descritas no Quadro 4.

Dianteda leitura overload, por conta da limitação técnica do aparelho, foi considerado o

Quadro 4. M édias dos resultados encontrados no experimento.

\begin{tabular}{|c|c|c|c|c|}
\hline \multirow{2}{*}{ Arma } & \multicolumn{3}{|c|}{ Local } \\
\cline { 2 - 5 } & \multicolumn{2}{|c|}{ Estande Campo aberto } \\
\cline { 2 - 5 } & $\mathrm{Np} \mathrm{em} \mathrm{dB}(\mathrm{A})$ & $\mathrm{Np} \mathrm{em} \mathrm{dB}(\mathrm{C})$ & $\mathrm{Np} \mathrm{em} \mathrm{dB}(\mathrm{A})$ & $\mathrm{Np} \mathrm{em} \mathrm{dB}(\mathrm{c})$ \\
\hline \multirow{2}{*}{ Fuzil (FAL) 7,62mm M 964} & $146,9 \pm 0,15$ & $146,1 \pm 0,4$ & $147,0 \pm 0,3$ & $147,2 \pm 0,0$ \\
\hline
\end{tabular}

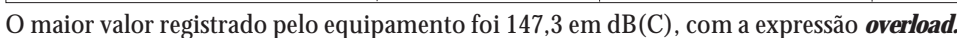

$\mathrm{Np}=$ Nível de pico 
maior nível de pressão sonora registrado ( 147.3 $\mathrm{dB}(\mathrm{C})$ ) e também considerado como valor de atenuação (NRRsf) a intensidade de 12dB (mé todo B - M étodo do Ouvido Real).

Neste trabalho, os critérios e a fórmula da Norma Técnica da Fundacentro ${ }^{9}$ foram utilizados para o cálculo da quantidade de pulsos de impacto a que o trabal hador pode estar exposto de acordo com a magnitude do pulso, com esem presença da proteção auditiva oferecida pelo modelo tipo plug da 3M.

\section{Discussão}

A metodologia utilizada para o cál culo da quantidade de pulsos de impacto a que o trabalhador pode ficar exposto em cada jornada de trabalho foi baseada na fórmula da Norma Técnica da Fundacentro $0^{9}$ e mostrou-se adequada tanto por sua representatividade como instrumento deprevenção, uma vez quea NR 15 restringe-sea crité rios meramente legai $s^{27}$, como também por seguir as orientações do NIOSH para a medição técnica do ruído de impacto.

A opção pela análise através do método $B$ (norma ANSI 12.6/1997 - Método do Ouvido Real), em que no seu experimento é valorada a colocação do protetor pelo próprio ouvinte, ocorreu em função de que os valores de atenuação, desvio padrão e o nível real de redução de ruído do protetor estão o mais próximo possível dos valores obtidos no mundo real e não são apenas representativos de uma situação teste-laboratorial.

Aplicando-se a fórmula da $\mathrm{NHO} 01, \mathrm{~Np}=$ $160-10$ Log $n$; onde $N p=$ Nível de pico e $n=$ número de pulsos, numa jornada de trabalho, temos:

. Para osmilitares sem proteção auditiva: 147,3 $=160-10 \log n=>\log n=1,27=>n=18,62$ tiros

. Para osmilitares com proteção auditiva com NRRsf de 12 dB: 147,3 - 12 (atenuação) = 160 $10 \log n=>\log n=2,47=>n=295,12$ tiros

Realizando esse cálculo com os valores referidos pela Revista Brasileira de Otorrinolaringologia (RBORL), que atribui ao rifle automático, classificação genérica do FAL, o N PS de 171 dB, ter-seá:

- Para os militares sem proteção auditiva: 171 $=160-10 \log n=>\log n=-1,1=>n=0,08$ tiros

- Para os militares com proteção auditiva com NRRsf de $12 \mathrm{~dB}: 171-12$ (atenuação) $=160-10$ $\log n=>\log n=0,1=>n=1,26$ tiros
A execução deste experimento sejustifica por não ter sido encontrado, em nenhuma das referências citadas, processo de medição que estabeleça o nível de pressão sonora e a quantidade de pulsos de impacto diante do uso de protetor auditivo do tipo plug com nível real de redução de ruído (NRRsf) de 12dB.

0 nível de pressão sonora recebido na atividade pesquisada, mesmo com o protetor auditivo tipo plug da 3M , modelo 1100, é, com certeza, algo acima de $135.3 \mathrm{~dB}(\mathrm{C})$, o que é suficiente, segundo a N R 15, para oferecer risco grave eiminente.

Obtivemos um intervalo entre 1.750 e 2.900 pulsos diários durante as atividades de tiro que foram acompanhadas nos quatro dias de atividade, distribuídos nos cinco estandes. Ressaltase que, nestas atividades, os instrutores eram os mesmos durante toda a jornada, ou seja, estes profissionais estiveram expostos a todos os impulsos, posicionados imediatamente atrás dos atiradores.

Já os instruendos, que estavam esperando a vez na linha de tiro, permaneceram entre dez e quinze metros atrás da linha de tiro, o que atenua o nível depressão sonora recebido durantea espera; porém, os instruendos que estão na linha de tiro durante os seus disparos estão expostos aosimpulsos detoda a linha detiro, o que variou de dez a dezesseis atiradores, com distância de $0,60 \mathrm{~m}$ a $1,20 \mathrm{~m}$ entre eles. Isso significa al go entre 250 e 350 impulsos, só na sua série de tiro.

Durante as entrevistas, cujo objetivo era mapear e entender a dinâmica desses exercícios de tiro, foi possível constatar dois grupos distintos de informantes que afirmavam não utilizar a proteção auditiva: o primeiro grupo não usa por não possuir o equipamento de proteção auditiva, que deveria ser distribuído pelo Exército; 0 segundo grupo apresentava argumentos ligados à não identificação do risco auditivo como: "não vejo necessidade e o barulho não me incomoda"; "por que o barulho do tiro é muito vibrante"; e "não quis usar ou não tive vontade", ou ainda, "não gosto!".

Já os militares que utilizavam proteção auditiva o faziam basicamente por dois motivos: 0 desconforto dos ruídos de impacto e a prevenção de futuros problemas auditivos. Esse comportamento denota, de certa forma, uma capacidade de associar uma situação de risco a futuros efeitos danosos à saúde.

A literatura consultada evidenciou que, quando o ouvido humano é exposto ao ruído de impacto, com intensidade sonora da ordem de 120 
dB ou superior, ocorre trauma acústico, desencadeado por ação mecânica de caráter irreversível epermanente. Os níveis produzidos pelo $\mathrm{Fu}$ zil Automático Leve (FAL) calibre 7,62mm que foram encontrados neste trabal ho ultrapassaram $135 \mathrm{~dB}(\mathrm{C})$ e, portanto, são indicadores de risco iminente de perda auditiva.

O NIOSH recomenda que, para níveis de exposição maiores que $100 \mathrm{~dB}(\mathrm{~A})$, deve-se recorrer ao uso da dupla proteção, apesar de reconhecer que a atenuação aumenta em apenas 5 a 10 $\mathrm{dB}(\mathrm{A})^{22}$.

N este estudo, os níveis encontrados ultrapassaram $135 \mathrm{~dB}$ (C). 0 protetor utilizado foi um modelo de inserção, descartável e que, segundo os critérios estabelecidos na norma técnica especializada, apresentou o valor deatenuação (NRRsf) igual a $12 \mathrm{~dB}(\mathrm{~A})$.

0 ruído deimpacto encontrado lesa irreversivelmente as estruturas cocleares, causando lesões auditivas, extra-auditivas e agravos psicossociais. As entrevistas demonstraram que os danos, suas consequências e riscos não são percebidos pelos instruendos e tampouco pelos instrutores, o que pode, no futuro, vir a comprometer a entrada no mercado de trabalho, por serem estes portadores de perda auditiva induzida por ruído.

\section{Considerações finais}

Os resultados finais sobre a efetividade da proteção auditiva utilizada pelos militares do Exército Brasileiro em presença deruído deimpacto igual ou superior a um nível de pressão (NPS) de 135 $\mathrm{dB}$, decorrente da atividade de tiro de instrução básico (TIB), reforçam as orientações do NIOSH de que outras variáveis têm influência nos efeitos ao aparelho auditivo, como, por exemplo, amplitude, duração, tempo de subida, número de impactos, taxa de repetição e fator de crista e que os efeitos aditivos e sinérgicos também precisam ser considerados. Reforça-se, assim, a necessidade do desenvolvimento de pesquisas sobre ruído de impacto, danos auditivos, agravos extra-auditivos e psicossociais, além da caracterização sobre a real efetividade da atenuação que é oferecida pelo protetor auditivo.
A escolha ou indicação do protetor auditivo, principalmente em presença de ruído de impacto, deveser criteriosa econsiderar aspectos técnicos como valores de atenuação, desvio padrão, nível de redução de ruído (NRR), nível real de redução de ruído (NRRsf); portanto, obedece a critérios técnicos e não pode ser através da livre escolha do usuário. A opção, para este tipo de exposição, deveser por protetores auditivos tipo concha, não lineares ou dotados de redução ativa de ruído.

A NR7 do M inistério do Trabalho e Emprego preconiza que, em presença do agente físico ruído, há obrigatoriedade de implantação e implementação de Programas de Conservação de Audição, como forma de prevenção. 0 Exército Brasileiro segueuma legislação diferenciada, mas não possui um programa de prevenção de perda auditiva para seus militares.

Diantedas análises realizadas, entende-seque a real redução do ruído, oferecida pelo protetor modelo 1100 da 3M , como alternativa de segurança auditiva, corresponde ao valor de atenuação NRRsf de 12 dB. Este valor não caracteriza efetividade na proteção auditiva, não obedece a critérios técnicos e não pode ser indicado para proteção ao ruído de impacto.

No caso dos militares que fizeram uso de algodão como tentativa de proteção auditiva durante o TIB, não foi possível calcular nenhum valor de atenuação, por conta das características físicas oferecidas por este material e pela ausência de descrição científica e experimentos sobre efetividade de atenuação auditiva.

Os resultados obtidos no presenteestudo vão ao encontro dos resultados do estudo realizado por Silva et al. ${ }^{9}$ com 97 militares, que além de identificar o prejuízo auditivo irreversível, ressalta a importância da adoção de medidas preventivas ao dano auditivo nesses profissionais. Além destas ações, e diante das possibilidades de implicações legais decorrentes da legislação brasileira existente, há necessi dade de que o Exército forneça esclarecimentos aos instruendos e instrutores sobre a correta utilização e efetividade do uso do protetor auditivo para esta categoria profissional. 


\section{Colaboradores}

EB N eves trabal hou na concepção, procedimentos metodológicos e interpretação dos dados; M Soalheiro trabalhou na revisão crítica e na redação final.

\section{Agradecimentos}

Ao Doutor M arco N abuco, da Divisão M etrologiaAcústica edeVibrações do Inmetro, pelo apoio na medição dos níveis de pressão sonora durante a realização do tiro defuzil.

\section{Referências}

1. Medeiros LB. Ruído: efeitos extra-auditivos no corpo humano [monografia]. Porto Alegre (RS): Centro de Especialização em Fonoaudiologia Clínica e Audiologia Clínica; 1999.

2. Bento RF, M initi A, Marone SAM. Tratado de otologia. São Paulo: Editora USP; 1998. p. 274-282.

3. Brasil. Portaria no 19, de 9 de abril de 1998- Anexo 1 - Diretrizes e Parâmetros Mínimos para Avaliação e Acompanhamento da Audição de Trabalhadores Expostos a Níveis de Pressão Sonora Elevados. Diário Oficial da União 1998, 22 abr.

4. Oliveira JAA. Fisiologia Clínica da Audição. In: Nudelmann AA, Costa EA, Seligmann J, Ibañez RN, organizadores. PAIR: Perda Auditiva Induzida pelo Ruído. Porto Alegre: Bagaggem Comunicações Ltda; 1997. p. 101-140.

5. Costa EA, Morata TC, Kitamura S. Patologia do ouvido relacionada com o trabalho. In: Mendes $R$, organizador. Patologia do trabalho. V. 2. 2a ed. São Paulo: Atheneu; 2003. p. 1253-1282.

6. Brasil. Portaria no 3.214, de 08 de junho de 1978. Aprova as Normas Regulamentadoras - NR - do Capítulo V, Título II, da Consolidação das Leis do Trabalho, relativas à Segurança e M edicina do Trabalho. Diário Oficial da União 1978; 6 jul.

7. National Institute for Occupational Safety and $\mathrm{H}$ ealth. Criteria for a Recommended Standard-O ccupational Noise Exposure. Revised Criteria a e b. Cincinnati: NIOSH; 1998.

8. Mendes $\mathrm{R}$, organizador. Patologia do trabalho. Vol. 1. 2a edição. São Paulo: Atheneu; 2003.

9. Silva AP, Costa EA, Rodrigues SMM, Souza HLR, M assafera VG. Audiometric assessment for military personnel. Rev. Bras. Otorrinolaringol [periódico na Internet] 2004 [acessado 2006 out 02];70(3): [cerca de 7 p.]. Disponível em: http://www.scielo.br/ scielo.php?script $=$ sci_arttext $\&$ pid $=$ S0034$72992004000300010 \&$ Ing $=$ =en\& $\mathrm{nrm}=\mathrm{iso}$

10. Fundacentro. NHO 01 - Norma de Higiene Ocupacional Procedimento Técnico - Avaliação da Exposição O cupacional ao Ruído. [site da Internet] [acessado 2006 set 25]. Disponível em: http://www. fundacentro.gov.br/CTN/NH O 01.pdf

11. Intensidade do Ruído em Armas de Guerra. Revista Brasileira de Otorrinolaringologia [periódico na Internet] [acessado 2006 out 05]. Disponível em: http://www.rborl.org.br/conteudo/acervo/acervo. asp?id $=942$

12. Godoy TCM. Perdas auditivas induzidas pelo ruído em militares: um enfoque preventivo [dissertação]. São Paulo (SP): PUC; 1991

13. Seballos SL. Condição auditiva de praticantes com arma de fogo [dissertação]. Santa M aria (RS): Universidade Federal de Santa M aria; 1995.

14. Neves-Pinto RM N, M onteiro ARC, Selimam J. Perda auditiva induzida pelo ruído: revisão das Publicações por Brasileiros no período 1938-1970. In: Nudelmann AA, Costa EA, Seligmann J, Ibañez RN, organizadores. PAIR - Perda auditiva Induzida pelo Ruído. Porto Alegre: Bagaggem Comunicação; 1997. p. 33-39.

15. Brito NAL. Limiares auditivos na frequência de $12.000 \mathrm{~Hz}$ em Aeronavegantes. Revista de M edicina Aeronáutica Brasileira 1998; 48:65-67. 
16. Uchimura KY, Bosi MLM. Quality and subjectivity in the evaluation of health services and programs. Cad Saude Publica [periódico na Internet] 2002 [acessado 2006 mar 15]; 18(6):[cerca de 10 p.]. Disponível em: http://www.scielosp.org/scielo.php?script=sci 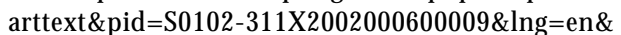 $\mathrm{nrm}=\mathrm{iso}$

17. M inayo M CS. Pesquisa social: teoria, método e criatividade. 19a ed. Petrópolis: Vozes; 2001.

18. Lincoln YS, Guba EG. Naturalistic inquiry. Beverly Hills: Sage; 1985.

19. Brasil. Estado-M aior do Exército. Instruções Gerais de Tiro com o Armamento do Exército - IGTAEx. Brasília: EGGCF; 2001.

20. Gerges SNY. Ruído: Fundamentos e Controle. $2^{a}$ ed. Florianópolis: NR Editora; 2000.

21. Berger EH. In: Mendes R, organizador. Patologia do trabalho. V. 1. 2ª edição. São Paulo: Atheneu; 2003.

22. National Institute for Occupational Safety and Health. In: M endes R, organizador. Patologia do trabalho. V. 1. 2a edição. São Paulo: Atheneu; 2003.

23. Damongeot EB. In: M endes $\mathrm{R}$, organizador. Patologia do trabalho. V. 1. 2ª edição. São Paulo: Atheneu; 2003.

24. 3M do Brasil. Boletim técnico dos protetores auditivos modelos 1100 e 1110. [site da Internet]. [acessado 2006 set 25]. Disponível em: http://www.3m. com/ intl/br/mineracao/download/1100\%201110. pdf

25. Brasil. Lei $n^{\circ} 6.880$, de 9 de dezembro de 1980. Dispõe sobre o Estatuto dos M ilitares. Diário Oficial da União 1980; 11 dez.

26. Comando de Operações Terrestres. Caderno de Instrução $\mathrm{Cl}$ 32/2: gerenciamento de risco aplicado às atividades M ilitares. 1a ed. Brasília: EGGCF; 2005.

27. Nepomuceno JA. Avaliação da exposição ao ruído. In: Nudelmann AA, Costa EA, Seligmann J, Ibañez RN, organizadores. PAIR: Perda auditiva induzida por ruído. Porto Alegre: Bagagem Comunicação; 1977. p.77-79.

Artigo apresentado em 26/03/2007

Aprovado em 31/05/2007

Versão final apresentada em 15/06/2007 